\title{
A review of the New World species of the parasitoid wasp Iconella (Hymenoptera, Braconidae, Microgastrinae)
}

\author{
José L. Fernández-Trianal,2,†, Sophie Cardinal ${ }^{2, \ddagger}$, James B. Whitfield ${ }^{3, \S}$, \\ Winnie Hallwachs ${ }^{4, I}$, M. Alex Smith ${ }^{1,5, \pi}$, Daniel H. Janzenr ${ }^{4, \#}$
}

I Biodiversity Institute of Ontario, University of Guelph, Guelph, ON N1G 2W1 Canada 2 Canadian National Collection of Insects, Agriculture and Agri-Food Canada, 960 Carling Ave., Ottawa, ON, K1A OC6 Canada 3 Department of Entomology, University of Illinois, Urbana, IL 61801 USA 4 Department of Biology, University of Pennsylvania, Philadelphia, PA 19104-6018 USA 5 Department of Integrative Biology, University of Guelph, Guelph, ON, N1G 2W1 Canada

\section{† http://zoobank.org/4469D91F-BBC1-4CBF-8263-EBFE2A95E4BF \\ ‡ http://zoobank.org/9D43F9ED-9B25-4C55-A99E-84D62F7204CO \\ § http://zoobank.org/7A98AB5F-552D-4437-8F5D-C593CA713506 \\ | http://zoobank.org/B2F86601-DF32-469C-942F-11116282C467 \\ I http://zoobank.org/E46EE6EB-E096-4FCD-BF5A-F91D4A8294EE \\ \# http://zoobank.org/C57962C8-05DD-422C-AC8C-786C16897EFB}

Corresponding author: José L. Fernández-Triana (jftriana@uoguelph.ca)

Academic editor: K. van Achterberg | Received 18 March 2013 | Accepted 2 July 2013 | Published 7 August 2013

http:/zoobank.org/6852A1D-A2C4-4328-B057-F2AF41829C5A

Citation: Fernández-Triana JL, Cardinal S, Whitfield JB, Hallwachs W, Smith MA, Janzen DH (2013) A review of the New World species of the parasitoid wasp Iconella (Hymenoptera, Braconidae, Microgastrinae). ZooKeys 321: 65-87. doi: $10.3897 /$ zookeys.321.5160

\begin{abstract}
The New World species of Iconella (Hymenoptera: Braconidae, Microgastrinae) are revised. Iconella andydeansi Fernández-Triana, sp. n., I. canadensis Fernández-Triana, sp. n., and I. jayjayrodriguezae Fernández-Triana, sp. n., are described as new. Iconella isolata (Muesebeck, 1955), stat. r., previously considered as a subspecies of I. etiellae (Viereck, 1911), is here elevated to species rank. All species have different, well defined geographic distributions and hosts. Taxonomic keys are presented in two formats: traditional dichotomous hardcopy versions and links to electronic interactive versions (software Lucid 3.5). Numerous illustrations, computer-generated descriptions, distributional information, host records (mostly Lepidoptera: Crambidae
\end{abstract}

Copyright José L. Fernández-Triana et al. This is an open access article distributed under the terms of the Creative Commons Attribution License 3.0 (CC-BY), which permits unrestricted use, distribution, and reproduction in any medium, provided the original author and source are credited. 
and Pyralidae), and DNA barcodes (where available) are presented for every species. Phylogenetic analyses of the barcoding region of COI indicate the possibility that Iconella is not monophyletic and that the New World species may not form a monophyletic group; more data is needed to resolve this issue.

\section{Keywords}

Iconella, Microgastrinae, New World, taxonomic review, host caterpillars, DNA barcoding, Area de Conservacion Guanacaste, parasitoid wasps

\section{Introduction}

The genus Iconella was erected by Mason (1981) to accommodate a group of Apanteles species with a sinuated vein cu-a in the hind wing, a character he interpreted as plesiomorphic among Microgastrinae. Iconella includes all species of Nixon's sundanus-group and part of the merula-group (Nixon 1965, Whitfield 1997). It is cosmopolitan, with 33 described species, most of them found in the Palearctic (Yu et al. 2012). All species with known hosts are solitary parasitoids of microlepidoptera, especially concealed feeders (Whitfield 1997); one species, Iconella isolata (Muesebeck, 1955) has been extensively studied in the tropics for biological control of a pest caterpillar (e.g. Bennett 1960, Bartlett et al. 1978).

Until now, only one species was known from the New World, Iconella etiella (Viereck, 1911). However, ongoing research on the Microgastrinae fauna of Area de Conservacion de Guanacaste (ACG) in northwestern Costa Rica (Smith et al. 2008, Janzen et al. 2009) and study of specimens housed in the Canadian National Collection of Insects (CNC) in Ottawa, Canada, have both revealed several new species for the Western Hemisphere. They are described here, and a key to all species in the New World is provided.

It should be noted that Achterberg (2002) did not consider Iconella as a valid genus and transferred its species (as well as those of other genera) back to Apanteles. However, that decision has not been universally accepted (e.g. Fernández-Triana 2010, Broad et al. 2012) and is still a subject of debate. In this paper we treat Iconella as a valid genus on its own.

\section{Methods}

Iconella is rare in collections, and even when present, specimens tend to be categorized as "unidentified Microgastrinae". In the New World, with the exception of one species, the genus seems to be rare in nature as well. Even long-term and comprehensive rearing programs -such as those in ACG, Costa Rica- have recovered relatively few specimens. This study is mostly based on the examination of unidentified Iconella specimens housed in the $\mathrm{CNC}$, representing close to 40 specimens; 11 specimens reared by the ACG inventory -and housed in the CNC and the Illinois Natural History Survey, Champaign, Illinois, United States (INHS); one specimen from the Laurentian Forestry Center, Ste.-Foy, Quebec, Canada (LFS); and the holotype of I. etiellae from the Smithsonian Institution, Washington DC, United States (NMNH). 
Morphological terms and measurements of structures are mostly as used by Mason (1981), Huber and Sharkey (1993) and Whitfield (1997). Non-morphological characters are also provided in the key whenever available (e.g., host species, geographical distribution). Those features are included in brackets at the end of the corresponding couplet and are intended as supplementary information that can help with identification.

The species descriptions are based mostly on the holotype female, with other specimens studied (when available) for intraspecific variation. When the holotype could not be examined (I. isolata) or it was a male (I. etiellae), female specimens were used for these redescriptions.

Lucid 3.5.4 (http://www.lucidcentral.com/) software was used to generate automatic descriptions of the species and to prepare Lucid identification keys. A dataset of 20 characters and 126 character-states was used to provide uniform descriptions for all species treated. Description format includes one sentence per character, with the character (in italics) mentioned first and the character-state following after a colon, e.g., "Pterostigma color: mostly brown, with yellowish-white spot at anterior $0.2 \times$ ".

A map with the distribution of all New World species of Iconella was generated using SimpleMappr (Shorthouse 2010).

In the taxonomic treatment of species, "Specimens Examined" presents the specimen's information in the following format: "Number of females/males, acronym of the storing institution between parentheses, COUNTRY: State/Province, city, other locality details, coordinates (in Decimal Degrees, abbreviated as "Lat:" and "Long:"), date, collector name, biological information on host (starting with "ex"), ACG database codes (in the format "yy-SRNP-xxxxxx" or "DHJPARxxxxxxx", with the former being the voucher code of the host and the latter being the voucher code of the parasite). For states of the United States and for Canadian provinces/territories, acronyms consisting of two capital letters are used, following Canada Post (http://www.canadapost.ca/tools/pg/manual/PGaddress-e.asp).

DNA barcodes for all specimens that were barcoded were obtained using DNA extracts prepared from single legs using a glass fibre protocol (Ivanova et al. 2006). Extracts were re-suspended in $40 \mu \mathrm{l}$ of $\mathrm{dH} 2 \mathrm{O}$, and a 658-bp region near the 5' terminus of the COI gene was amplified using standard primers (LepF1-LepR1) following established protocols (Smith et al. 2006, 2007, 2008). If the initial 658 bp amplification was not successful, composite sequences were generated using internal primers (primers are as detailed in Smith et al. (2008)). All available DNA barcodes for Iconella specimens from the New World and the Palearctic are available on the Barcode of Life Data System (BOLD, www.boldsystems.org/), along with sequences from Apanteles and Dolichogenidea, which were used as outgroups in the phylogenetic analyses (dx.doi.org/10.5883/ DS-ASICON1). Collection information and accessions (BOLD and GenBank) for all sequences were already published in a previous paper (Smith et al. 2013).

DNA barcode sequences were aligned in Geneious Pro 6.0.5 (Drummond et al. 2011) using the default settings for a MUSCLE alignment. Because of the high AT content characteristic of Insect and Hymenoptera mitochondrial DNA, sequence divergences were calculated using the TN93model (Tamura and Nei 1993) and a neighbor-joining 
(NJ, Saitou and Nei 1987) tree of distances was generated using Geneious Pro 6.0.5 (Drummond et al. 2011) to provide a graphical representation of the species divergences.

Many of the Iconella specimens in BOLD were collected before 1979. Characteristic of such moderately-aged specimens, the COI fragments generated were less $(\sim 160 \mathrm{bp})$ than the full length characteristic of the standard DNA barcode ( $>600 \mathrm{bp})$ (Fernández-Triana et al. 2011). Therefore, more than half of the sequences used in the analyses described below were less than $300 \mathrm{bp}$, and $44 \%$ were less than $200 \mathrm{bp}$. Phylogenetic comparisons of fragments this small, and involving cases where there is even lower overlap will be compromised. However, small COI fragments $(<200 \mathrm{bp})$ are not devoid of phylogenetic signal and have been used to successfully identify species (Fernández-Triana 2010, Fernández-Triana et al. 2011, Hajibabaei et al. 2006).

To investigate the phylogenetic relationships of the species, the aligned dataset was analyzed in MrBayes v. 3.2.1 (Ronquist and Huelsenbeck 2003). Model selection was based on the Akaike Information Criterion as implemented in JModelTest v.2.1.1 (Darriba et al. 2012). Two independent analyses with 4 chains each were run in parallel for 10 million generations under a GTR $+\mathrm{I}+\mathrm{G}$ model. The parameter trace files of each run were observed in Tracer v.1.5 (Rambaut and Drummond 2009) to verify that the runs had converged on the same stationary distribution, and to decide on the appropriate number of generations to discard as burn-in. A 50\% majority rule consensus tree was constructed from the 18 million post-burn-in generations in Geneious Pro 6.0.5. The above protocol was followed for additional analyses in which 1 ) all $3^{\text {rd }}$ codon positions were removed from the dataset, 2) characters with more than $2 \%$ missing data were removed, and 3) sequences that were less than 547 base pairs long were eliminated. A Maximum Likelihood (ML) analysis was also run in RAXML v. 7.3.4 (Stamatakis 2006) under a GTR+I+G model. 1000 bootstrap (BS) replicates were run and the BS support values were then drawn onto the best-scoring ML tree. A parsimony analysis was conducted in TNT v.1.1 (Goloboff et al. 2008) using the "aquickie.run" script provided with the program.

\section{Results}

As with many other taxa, the generic status of Iconella will only be solved when a comprehensive phylogeny of Microgastrinae is carried out. In the meantime we think is best to keep it as a valid genus, based on the available morphological evidence. Mason (1981) already mentioned the sinuated vein cu-a in the hind wing as a plesiomorphic character that suggests the unique status of Iconella among similar genera. Besides that, we also consider the presence of a median longitudinal carina on the propodeum (or the secondary loss of that carina, which occurs in some Paleartic species but not in the New World species) as a strong support for the generic status of Iconella -in contrast with Apanteles, which (sensu Mason 1981) almost always has carinae defining a complete or partial areola on the propodeum, but never has a median longitudinal carina on the propodeum.

Phylogenetic analyses of the COI DNA, however, are inconclusive as to whether the genus Iconella is monophyletic (Figs 1 and 2). All Bayesian (only the tree resulting 


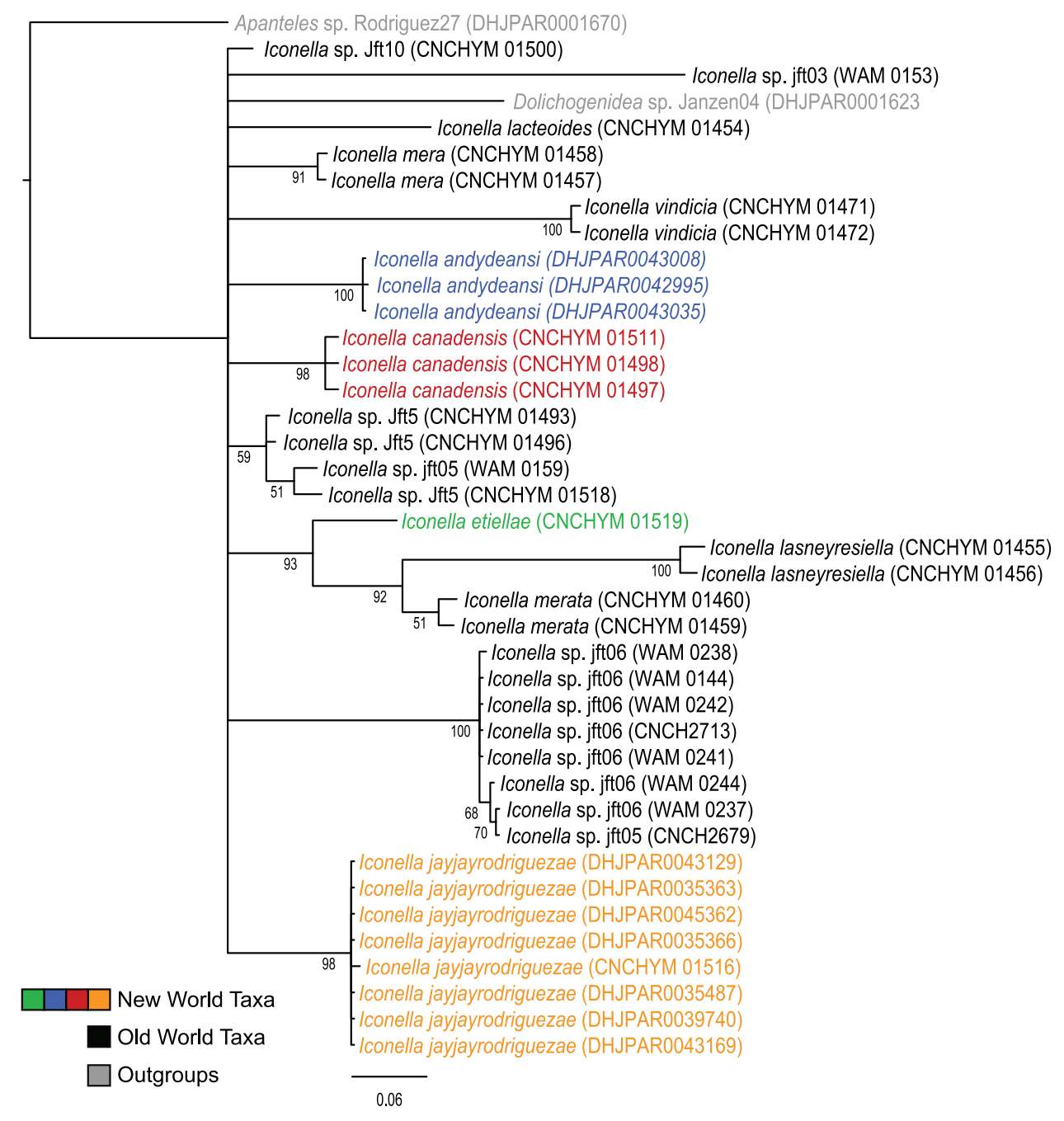

Figure I. Majority rule consensus tree calculated from the posterior tree samples of the Bayesian analysis of the full Iconella COI fragment dataset. Values below the branches are posterior probabilities. The New World species are individually color coded.

from analysis of the full dataset is shown in Fig. 1), ML (tree not shown), and parsimony (tree not shown) analyses failed to recover monophyly of the genus. However, the model-based methods do not offer strong support against monophyly either. The highest posterior probability (PP) supporting non-monophyly was 0.55 when $3^{\text {rd }}$ codon positions were removed from the Bayesian analysis, and BS support against monophyly in the ML analysis was only 0.21 .

We recognize five species of Iconella in the New World, including the three new species described in the present paper, and one that was previously considered as a subspecies (Muesebeck 1955) but is here elevated to species. We are also aware of 


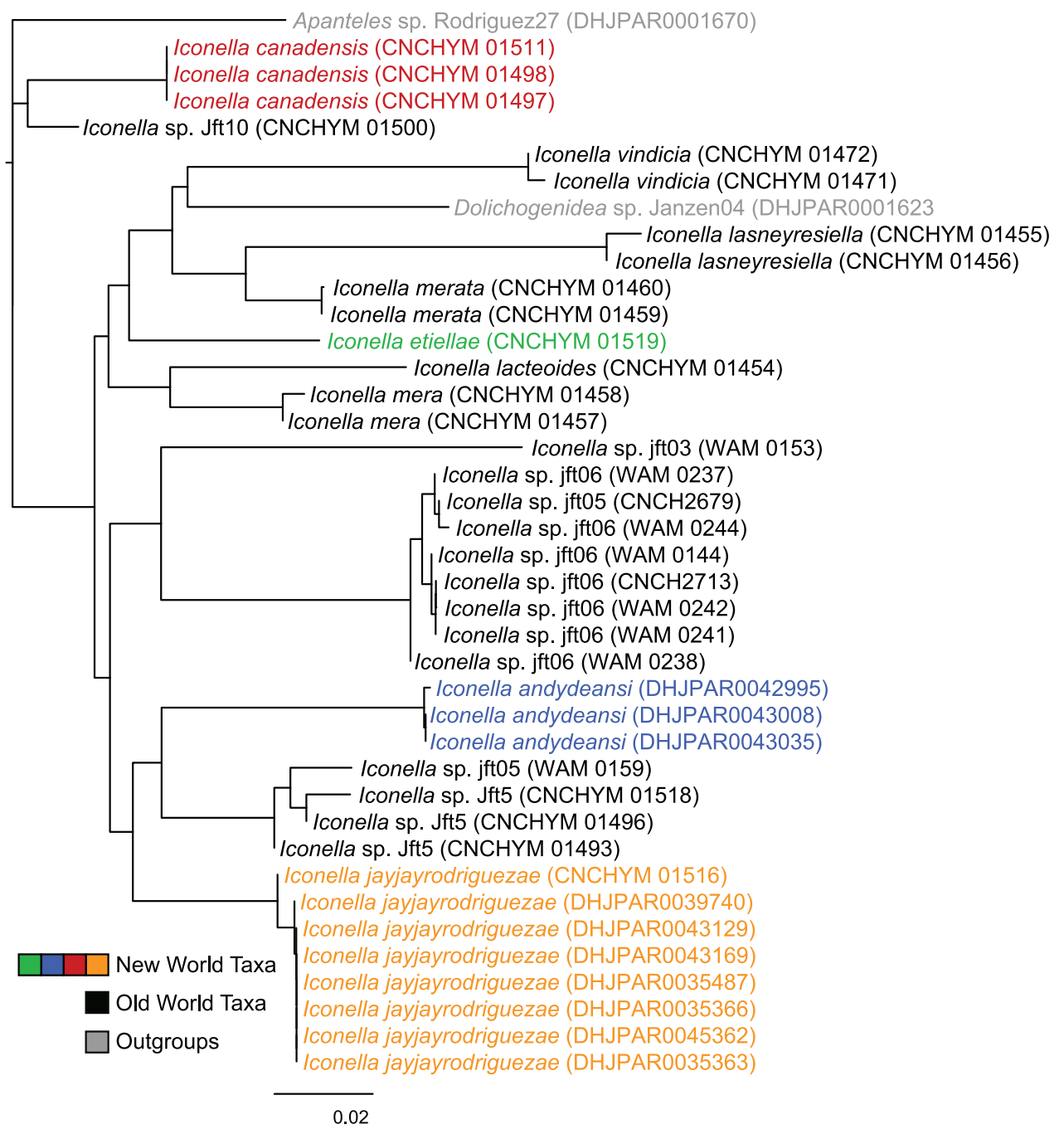

Figure 2. Neighbor-joining tree of the full Iconella COI fragment dataset using the TN93model. The New World species are individually color coded.

an additional sixth species, represented by two specimens in poor condition from British Columbia (Western Canada), deposited in the CNC. They were mentioned in Fernández-Triana (2010) as "Iconella sp. 2" and are different from all other New World species of the genus. However, until more material is found, it is impossible to describe that species.

All of the described species have a different, well delimited geographic range (Fig. 3) and also differ in their known hosts. Most of the host species in the Western Hemisphere belong to the Lepidoptera families Pyralidae and Crambidae (with appropriate caution that what was called Pyralidae in the past is in part called Crambidae at present). The NJ tree (Fig. 2) as well as Bayesian (Fig. 1), ML, and parsimony based 


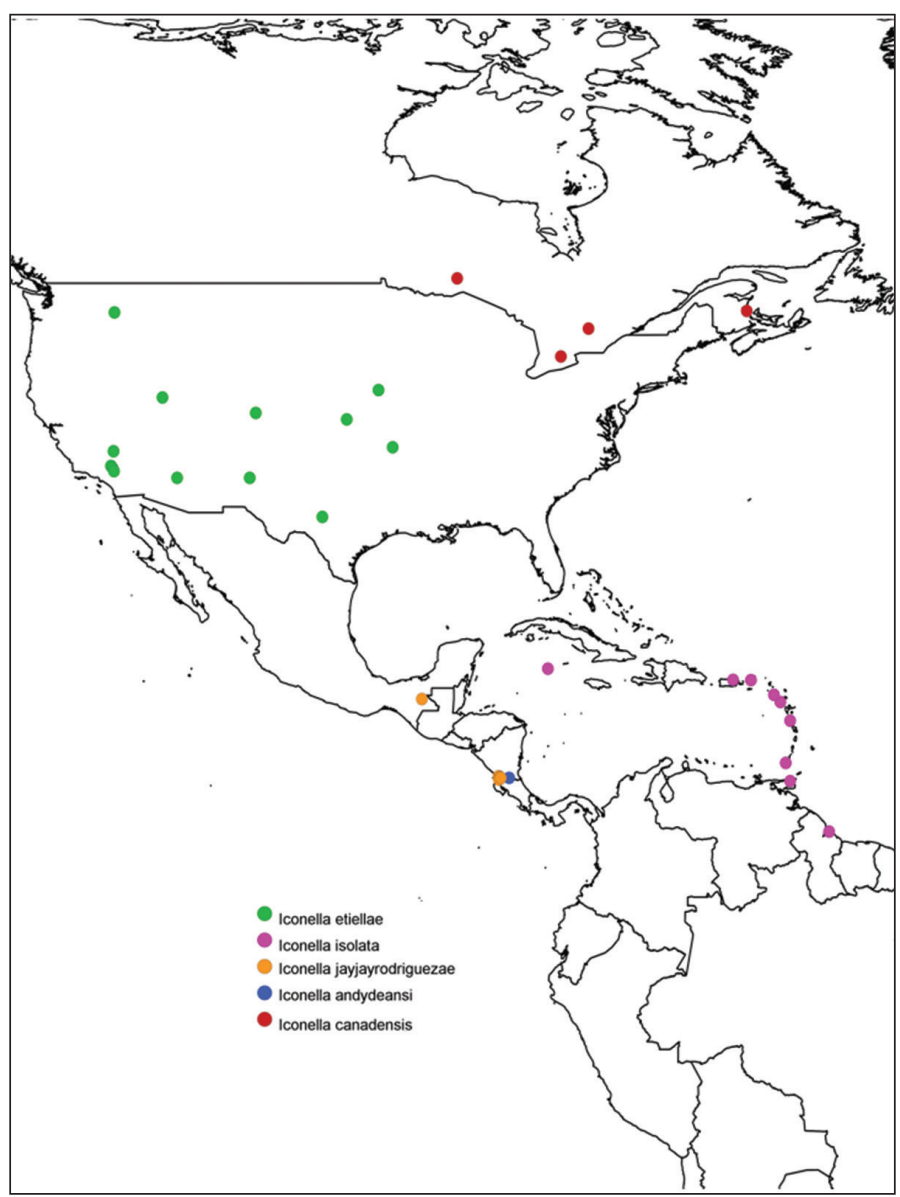

Figure 3. Distribution of New World species of Iconella. Species are individually color coded.

phylogenetic analyses of the barcodes support the validity of all New World species recognized herein (there is no molecular data available for $I$. isolata). However, the barcoding dataset did not contain a strong enough phylogenetic signal to resolve the phylogenetic relationships among Iconella species.

While these DNA barcodes and short COI fragments (mini-barcodes (Hajibabaei et al. 2006, Meusnier et al. 2008)) were sufficient to unambiguously separate the species of Iconella, more collections and longer DNA sequences will be needed to test the monophyly (or lack thereof) of Iconella.

\section{Key to the New World species of Iconella}

1 Propodeum mostly smooth and polished (as in Fig. 21), except for sparse punctures on the anterior $0.2 \times$ of propodeum, and rather small carinae ra- 
diating from median longitudinal carina; metatibia mostly yellow, at most with very small and faint brown spot on posterior 0.1 or less, and metatarsus mostly yellow, except for brown area on posterior half of first tarsomerus (Figs 17, 19, 23, 25); fore wing with most veins transparent or white, vein margins of same color than interior of vein (Figs 18, 24)....

Propodeum with anterior $0.2 \times$ covered with punctures, posterior 0.8 covered with mix of punctures, striated sculpture and carinae (as in Fig. 15); metatibia with brown to black coloration on posterior $0.2-0.4$, and metatarsus mostly dark brown, except for yellowish area on anterior half of first tarsomerus (as in Fig. 33); fore wing with at least some veins with thin brown margins and interior of veins yellow to light brown (Figs 5, 11,31).... 3 Pterostigma almost completely brown, with only small whitish spot anteriorly (Fig. 18); humeral complex half yellow, half brown; profemur almost completely dark brown (yellow area absent or limited to posterior 0.2 ); interocellar distance $2.4 \times$ or more posterior ocellus diameter (Fig. 20); mediotergite 2 width at posterior margin $4.6 \times$ or less its length (Fig. 22); larger species, body length (head to apex of metasoma) $3.0 \mathrm{~mm}$ or more, and fore wing length $3.3 \mathrm{~mm}$ or more. [Western and central United States: AR, AZ, CA, CO, IA, NM, OK, and WA. Hosts: Etiella zinckenella, Olycella junctolineella, Psorosina hammondi, and Ufa rubedinella (Pyralidae)]

Iconella etiellae (Viereck, 1911)

Pterostigma mostly transparent or whitish, with only thin brown margins (Fig. 24); humeral complex yellow to white; profemur mostly yellow, dark brown area limited to anterior 0.2 or less; interocellar distance $2.1 \times$ or less posterior ocellus diameter; mediotergite 2 width at posterior margin $5.0 \times$ or more its length (Fig. 26); smaller size, body length (head to apex of metasoma) $3.0 \mathrm{~mm}$ or less, and fore wing length $3.2 \mathrm{~mm}$ or less. [Caribbean islands and northern part of South America: British Virgin Islands, Cayman Islands, Dominica, Grenada, Guyana, Montserrat, Puerto Rico, Saint Kitts \& Nevis, Trinidad \& Tobago. Host: Ancylostomia stercorea (Pyralidae)]

Iconella isolata (Muesebeck, 1955), stat. r.

3- Ocular-ocellar line $1.6 \times$ posterior ocellus diameter; humeral complex half yellow, half brown; mediotergite 1 width at anterior margin $2.2 \times$ or less its width at posterior margin (Fig. 13); ovipositor sheaths length $0.8 \times$ or less metatibial length (Fig. 12); larger species, body length (head to apex of metasoma) $3.5 \mathrm{~mm}$ or more (rarely $3.2 \mathrm{~mm}$ ) and fore wing length $3.5 \mathrm{~mm}$ or more; an extra-tropical species distributed in North America north of $40^{\circ} \mathrm{N}$ (Canada). [Eastern Canada: NB, ON, and QC. Host: Epinotia solandriana (Tortricidae) and, likely, Acrobasis betulella (Pyralidae)]

..Iconella canadensis Fernández-Triana, sp. $\mathbf{n}$.

Ocular-ocellar line $2.0 \times$ or more posterior ocellus diameter; humeral complex fully yellow to white; mediotergite 1 width at anterior margin $3.1 \times$ or more its width at posterior margin (Figs 9, 36); ovipositor sheaths length 
$1.1 \times$ metatibial length (Figs 6, 32); smaller size, body length (head to apex of metasoma) $3.0 \mathrm{~mm}$ or less, and fore wing length $3.3 \mathrm{~mm}$ or less; tropical species from Central America south of $17^{\circ} \mathrm{N}$ (Mexico and Costa Rica) ...... 4

4 Profemur mostly yellow, dark brown area limited to anterior 0.2 or less; meso- and meta- femora mostly dark brown, with proximal $0.1-0.2$ yellow to orange; mesoscutellar disc sculpture centrally smooth with few, scattered punctures near margins (Fig. 36); mediotergite 2 width at posterior margin $4.1 \times$ or less its maximum length medially (Fig. 36); body length (head to apex of metasoma) $2.9-3.0 \mathrm{~mm}$; fore wing length 3.2-3.3 mm. [Costa Rica (ACG) and Mexico (Chiapas). Host: undescribed species of Phycitinae (Pyralidae)]

Iconella jayjayrodriguezae Fernández-Triana, sp. n.

- $\quad$ Profemur dark brown on anterior half, yellow on posterior half; meso- and meta- femora usually fully dark brown to black; mesoscutellar disc sculpture mostly with punctures scattered all over disc surface (Fig. 9); mediotergite 2 width at posterior margin $4.4 \times$ its maximum length medially (Fig. 9); body length (head to apex of metasoma) $2.7-2.8 \mathrm{~mm}$; fore wing length 3.0 $\mathrm{mm}$. [Costa Rica (ACG). Host: undescribed species of Spilomelinae (Crambidae)]

Iconella andydeansi Fernández-Triana, sp. $\mathrm{n}$.

\section{Taxonomic treatment of species, in alphabetical order}

\section{Iconella andydeansi Fernández-Triana, sp. $\mathbf{n}$.} http://zoobank.org/C9173C9E-1E3C-46A8-A459-97DDF2ABF1DF http://species-id.net/wiki/Iconella_andydeansi

Figures 4-9

Type locality. COSTA RICA, Alajuela, Area de Conservacion Guanacaste, Sector Rincon Rain Forest, Camino Rio Francia, 410m. Lat: 10.90425, Long: -85.28651.

Holotype. + , CNC. First label: DHJPAR0043035. Second label: Voucher: D.H.Janzen \& W.Hallwachs, DB: http://janzen.sas.upenn.edu, Area de Conservacion Guanacaste, COSTA RICA, 11-SRNP-41294. Collecting date of caterpillar host: 20.iii.2011, collection date (eclosion date) of wasp: 05.iv.2011.

Specimens examined. Paratypes: 1 ,, $1 \hat{O}$ (CNC) Costa Rica, same locality than holotype. Specimens voucher codes: DHJPAR0042995 and DHJPAR0043008.

Description. Promefur color: dark brown on anterior half, yellow on posterior half. Meso- and meta-femur color: fully dark brown to black (Fig. 4). Metatibia and metatarsus color: Metatibia with brown to black coloration on posterior $0.2-0.4 \times$; metatarsus mostly dark brown, except for yellowish area on anterior half of first tarsomerus (Fig. 6). Tegula and humeral complex color: tegula and humeral complex fully yellow to yellowish-white (Fig. 9). Pterostigma color: centrally yellow-white, with thin brown margins (Fig. 5). Fore wing veins color: at least some veins with 

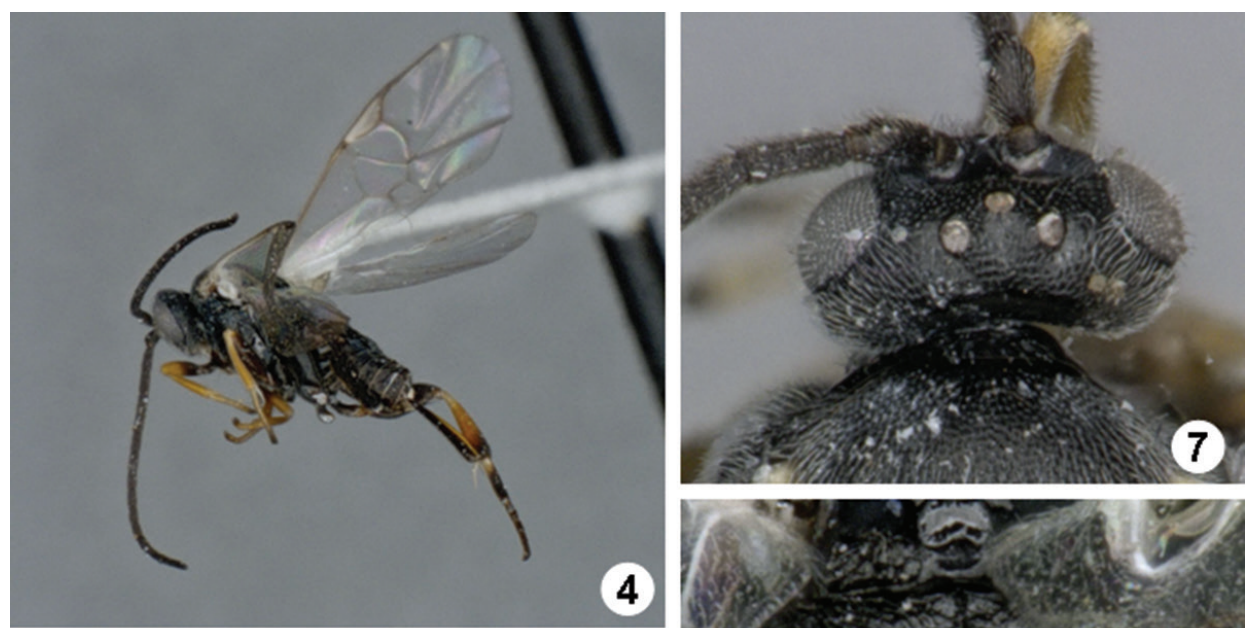

4
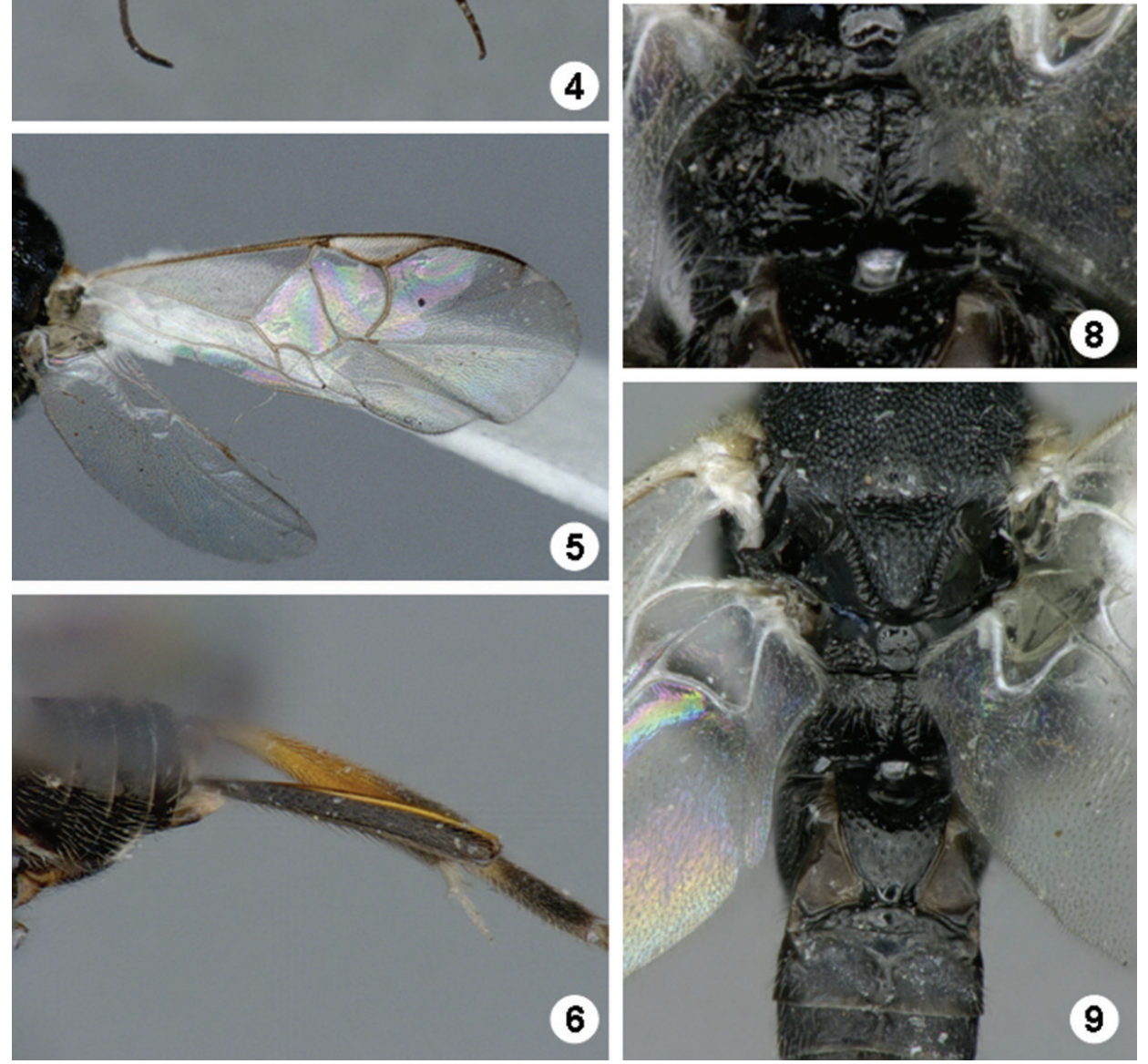

Figures 4-9. Iconella andydeansi. 4 Habitus, lateral view 5 Fore wing 6 Ovipositor sheats and metatibia 7 Head, dorsal view 8 Propodeum 9 Meso- and metasoma (partially), dorsal view.

thin brown margins and interior of veins yellow to light brown. Body length (head to apex of metasoma): $2.7 \mathrm{~mm}$ or $2.8 \mathrm{~mm}$. Fore wing length: $3.0 \mathrm{~mm}$. Ocular-ocellar linelposterior ocellus diameter: $2.2 \times$. Interocellar distance/posterior ocellus diameter: 
$2.1 \times$ (Fig. 7). Antennal flagellomere 2 length/width: $2.5 \times$ or $2.7 \times$. Antennal flagellomere 14 length/width: 1.3 $\times$. Length of flagellomere 2/length of flagellomere 14: $2.4 \times$. Metafemur length/width: $3.1 \times$. Mesoscutellar disc: mostly with punctures scattered all over disc surface (Fig. 9). Number of pits in scutoscutellar sulcus: usually 12 or less, ocasionally reaching up to 14 pits. Propodeum background sculpture: anterior 0.2$0.4 \times$ with rather dull puntures; posterior $0.6-0.8 \times$ mostly sculptured, with mix of small puntures and carinae (mostly radiating from strong, longitudinal median carina) (Fig. 8). Mediotergite 1 width at anterior margin/width at posterior margin: $3.4 \times$. Mediotergite 2 width at posterior margin/length: $4.4 \times$ (Fig. 9). Ovipositor sheaths length/metatibial length: $1.1 \times$ (Fig. 6).

Male. As female, although sculpture is slightly smoother.

Molecular data. We analyzed three full $658 \mathrm{bp}$ barcodes for this species.

Biology/ecology. Host: An undescribed species of Phycitinae (Pyralidae) with provisional name "phyjanzen021 Janzen855" in the ACG database (http://janzen.sas. upenn.edu/caterpillars/database.lasso). Caterpillar collected while feeding on the foliage of Lepidoploa salzmannii (Asteraceae).

Distribution. Only known from the holotype locality, in Sector Rincon Rain Forest of ACG at $410 \mathrm{~m}$.

Comments. The species has been reared only in one place from three caterpillars collected at the same time on the same species of food plant, out of $12,000+$ rearings of ACG Pyralidae of more than 200 species. A single additional specimen, identified by DNA barcoding, has been reared in 2012 from the same species of host caterpillar in the same place and on the same food plant, but was not available for study. Iconella andydeansi is sympatric with I. jayjayrodriguezae in ACG, the latter being a species with a slightly larger ACG rain forest distribution but equally narrow food plant record, and also known from Chiapas, Mexico. Those are the only two species of New World Iconella that are known to be sympatric, and that was revealed through extensive collecting in ACG and the use of DNA barcodes. It is likely that further collecting in other areas of the Neotropics, as well as the barcoding of more fresh specimens, will reveal additional species.

Etymology. This species is named in honor of Andy R. Deans (Pennsylvania State University, United States) in recognition of his major contribution to the taxonomy of the many species in the microgatrine genus Alphomelon that occur in Area de Conservación Guanacaste (e.g., Deans et al. 2003).

\section{Iconella canadensis Fernández-Triana, sp. n.} http://zoobank.org/C3E8D164-ABBC-4823-9D56-50E302065F55 http://species-id.net/wiki/Iconella_canadensis Figures 10-16

Type locality. CANADA. Ontario, Black Sturgeon Lake. Lat: 49.368333, Long: -88.881944 . 

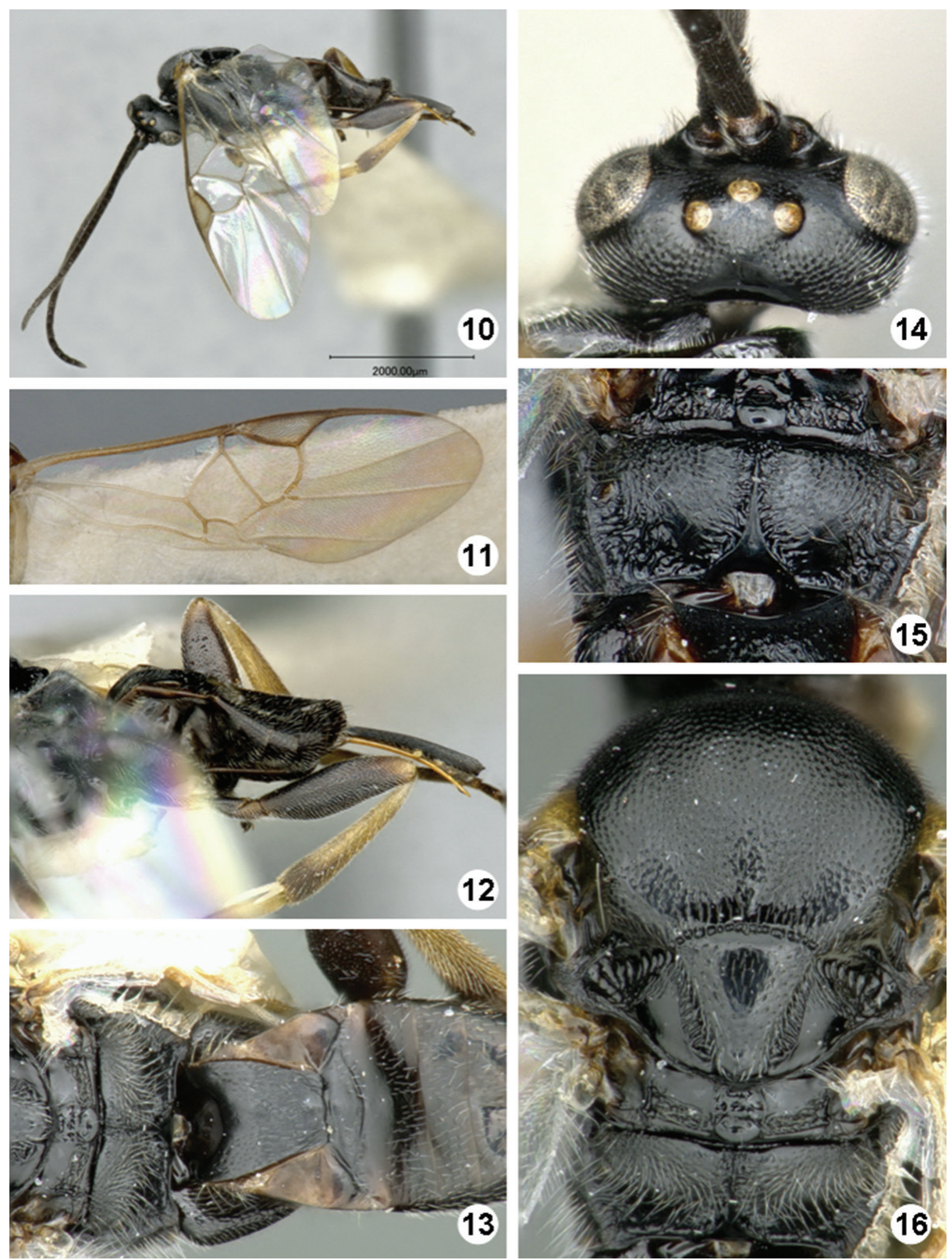

Figures 10-16. Iconella canadensis. I 0 Habitus, lateral view I I Fore wing $\mathbf{2}$ Ovipositor sheats, mesofermur, and metatibia 13 Propodeum, mediotergites 1-4, dorsal view 14 Head, dorsal view I5 Propodeum 16 Mesosoma, dorsal view.

Holotype. + , CNC. First label: Black Sturgeon Lake, Ontario, Em. 1-6-viii-1961, Insectary. Second label: Nest 97, Cell 4 ex provisions. Third label: W61319. Fourth label: Host either A. betullela or Rh. hasta. Fifth label: DNA Voucher CNCHYM 01498. 
Specimens examined. Paratypes: 3 q (CNC) Canada: ON, Black Sturgeon Lake, 21-29.vii.1961, 26.vii.1962, and 2.viii.1962, ex: Provisions Nests 52 and 66, one specimen with DNA Voucher CNCHYM01497; 1 \& (CNC) Canada: ON, Galt, 11.vii.1952; 1 q (CNC) Canada: ON, Whitney, 4.vii.1949, ex: Phalaenidae; 1 , 1 ô (CNC) Canada: NB, Kouchibouguac National Park, 30.viii.1967, code-6060B, DNA Voucher CNCHYM01511 and CNCHYM01512; 1 o (LFS) Canada: QC, Saint-Cléophas-de-Brandon, 4.vii.1968, ex: Epinotia solandriana on Betula papyrifera. Collecting dates of specimens examined: July and August (1949-1967).

Description. Promefur color: dark brown on anterior half, yellow on posterior half. Meso- and meta-femur color: mostly dark brown but with proximal $0.1-0.2 \times$ yellow to orange (Fig. 12). Metatibia and metatarsus color: Metatibia with brown to black coloration on posterior $0.2-0.4 \times$; metatarsus mostly dark brown, except for yellowish area on anterior half of first tarsomerus (Fig. 10). Tegula and humeral complex color: tegula and anterior half of humeral complex yellow to yellowish-white, posterior half of humeral complex light brown to dark brown. Pterostigma color: centrally yellow-white, with thin brown margins, rarely mostly brown, with yellowish-white spot at anterior $0.2 \times$ (Fig. 11). Fore wing veins color: at least some veins with thin brown margins and interior of veins yellow to light brown. Body length (head to apex of metasoma): $3.5 \mathrm{~mm}, 3.6 \mathrm{~mm}$, $3.7 \mathrm{~mm}$, rarely $3.2 \mathrm{~mm}$. Fore wing length: $3.8 \mathrm{~mm}, 3.9 \mathrm{~mm}, 4.0 \mathrm{~mm}, 4.1 \mathrm{~mm}$ or 4.2 $\mathrm{mm}$. Ocular-ocellar line/posterior ocellus diameter: $1.6 \times$. Interocellar distance/posterior ocellus diameter: $1.9 \times$ (Fig. 14). Antennal flagellomere 2 length/width: $3.0 \times$. Antennal flagellomere 14 length/width: $1.6 \times$. Length of flagellomere 2/length of flagellomere 14: $2.1 \times$. Metafemur length/width: $3.2 \times, 3.3 \times$, rarely $3.4 \times$. Mesoscutellar disc: mostly smooth with few, scattered punctures near margins (Fig. 16). Number of pits in scutoscutellar sulcus: usually 16, ocasionally only 14 pits. Propodeum background sculpture: anterior 0.2-0.4 $\times$ with rather dull puntures; posterior 0.6-0.8 $\times$ mostly sculptured, with mix of small puntures and carinae (mostly radiating from strong, longitudinal median carina) (Fig. 15). Mediotergite 1 width at anterior margin/width at posterior margin: $2.1 \times$ or $2.2 \times$. Mediotergite 2 width at posterior margin/length: $3.6 \times, 3.8 \times$ or $4.4 \times$ (Fig. 13). Ovipositor sheaths length/metatibial length: $0.7 \times$ or $0.8 \times$ (Fig. 12).

Male. As female.

Molecular data. We analyzed three short $164 \mathrm{bp}$ COI sequences from the DNA barcode region.

Biology/ecology. Host: Epinotia solandriana (Tortricidae) and likely Acrobasis betulella (Pyralidae) (see Comments below).

Distribution. Eastern Canada: NB, ON, QC.

Comments. The holotype has a label stating that it emerged from either Acrobasis betulella (Pyralidae) or Rheumaptera hasta (Geometridae). Based on the known biology of the genus Iconella in the world, the second alternative is unlikely, and thus we consider $A$. betulella as the potential host in that case. However, the pyralid host cannot be taken as definitive until more reared specimens confirm the decision.

Etymology. The name refers to the known distribution of the species, in Eastern Canada. 
Iconella etiellae (Viereck, 1911)

http://species-id.net/wiki/Iconella_etiellae

Figures $17-22$

Apanteles etiellae: Viereck 1911: 178.

Iconella etiellae (Viereck). Mason 1981: 75.

Type locality. UNITED STATES, Washington, Pullman.

Holotype. ${ }^{\lambda}$, NMNH (examined).

Specimens examined. 3 +, $3 \widehat{\diamond}$ (CNC) United States: CA (Apple Valley, Helendale, Kramer Hills and Panamint Valley) and UT (Dugway Proving Ground). Collecting dates of specimens examined: May and June (1953-1961).

Description. Promefur color: almost completely dark brown (yellow area absent or limited to posterior $0.2 \mathrm{x}$ ) (Fig. 17). Meso- and meta-femur color: mostly dark brown but with proximal 0.1-0.2 $\times$ yellow to orange (Fig. 19). Metatibia and metatarsus color: Metatibia mostly yellow, at most with very small and faint brown spot on posterior $0.1 \times$ or less; metatarsus mostly yellow, except for brown area on posterior half of first tarsomerus (Fig. 19). Tegula and humeral complex color: tegula and anterior half of humeral complex yellow to yellowish-white, posterior half of humeral complex light brown to dark brown. Pterostigma color: mostly brown, with yellowish-white spot at anterior $0.2 \times$ (Fig. 18). Fore wing veins color: most of veins transparent or at most yellowish-white, margins of same color than interior of vein. Body length (head to apex of metasoma): $3.0 \mathrm{~mm}$ or $3.1 \mathrm{~mm}$. Fore wing length: $3.3 \mathrm{~mm}$ or $3.4 \mathrm{~mm}$. Ocular-ocellar line/posterior ocellus diameter: $1.6 \times$ or $1.7 \times$. Interocellar distancelposterior ocellus diameter: $2.4 \times$ or $2.6 \times$ (Fig. 20). Antennal flagellomere 2 length/width: $2.6 \times$ or $2.7 \times$. Antennal flagellomere 14 length/width: $1.4 \times$ or $1.7 \times$. Length of flagellomere 2/length of flagellomere 14: $2.2 \times$. Metafemur length/width: $3.2 \times$ or $3.3 \times$. Mesoscutellar disc: mostly smooth with few, scattered punctures near margins (Fig. 22). Number of pits in scutoscutellar sulcus: usually 14 or more, ocasionally reaching up to 16 pits. Propodeum background sculpture: anterior $0.2-0.4 \times$ with fine puntures; posterior $0.6-0.8 \times$ mostly smooth, at most with some small carinae (mostly radiating from strong, longitudinal median carina) (Fig. 21). Mediotergite 1 width at anterior margin/width at posterior margin: $2.4 \times$ or $2.5 \times$. Mediotergite 2 width at posterior margin/length: $5.0 \times$ or $5.1 \times$ (Fig. 22). Ovipositor sheaths length/metatibial length: $1.1 \times($ Fig. 19).

Male. As female.

Molecular data. We analyzed one 607 bp barcode for this species.

Biology/ecology. Hosts: Etiella zinckenella, Olycella junctolineella, Psorosina hammondi, and Ufa rubedinella (Pyralidae).

Distribution. United States: AR, AZ, CA, CO, IA, NM, OK, WA.

Comments. There is a record from Virginia, United States (Yu et al. 2012). While we have not been able to examine that specimen, based in the known distribution of New World species of Iconella and their hosts, it is very likely that the VA specimen actually belongs to Iconella canadensis Fernández-Triana. 

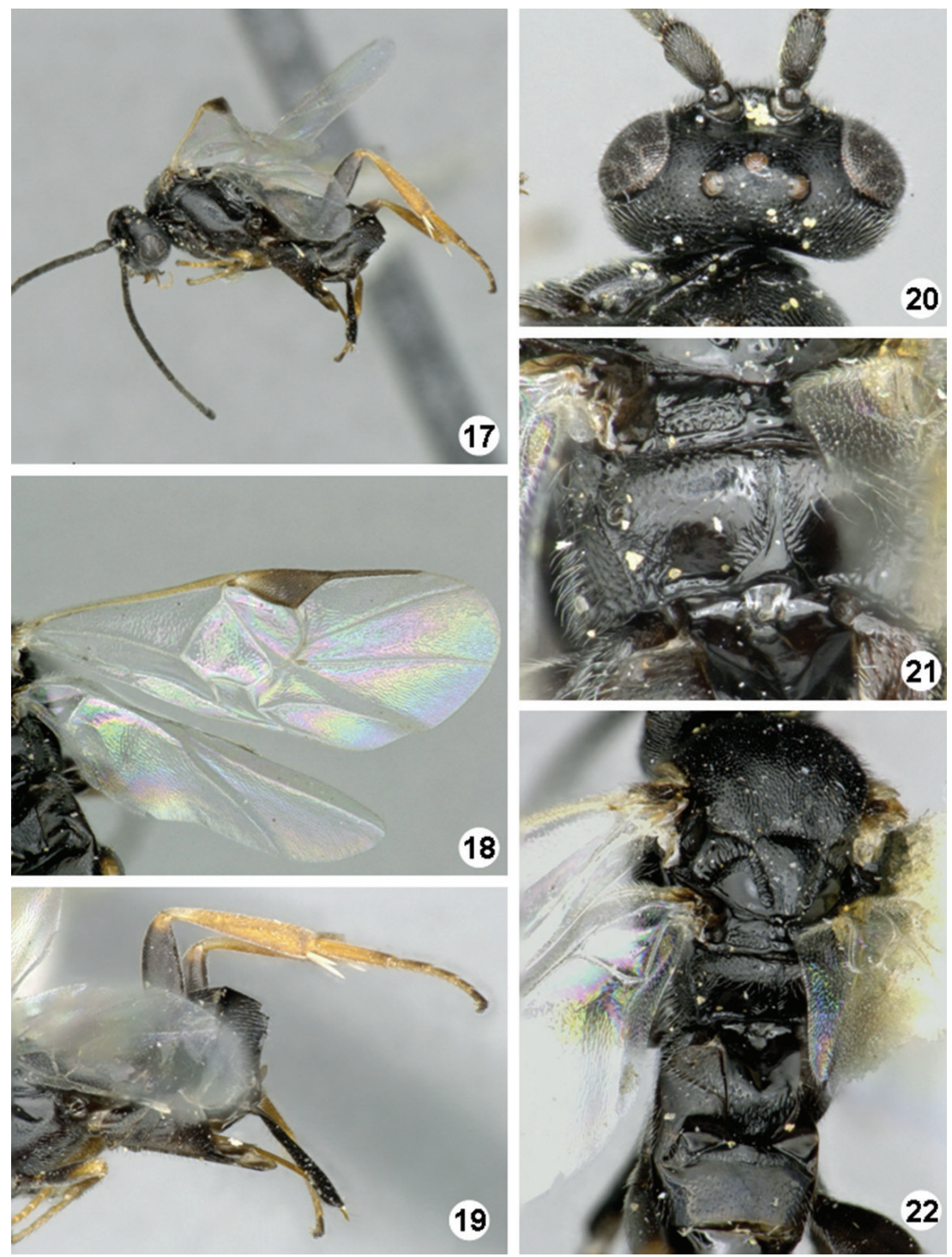

Figures 17-22. Iconella etiellae. 17 Habitus, lateral view 18 Fore wing 19 Ovipositor sheats, mesofermur, and metatibia 20 Head, dorsal view 2 I Propodeum 22 Meso- and metasoma (partially), dorsal view.

Iconella isolata (Muesebeck, 1955), stat. r. http://species-id.net/wiki/Iconella_isolata Figures 23-29

Apanteles etiellae isolatus Muesebeck, 1955: 161.

Type locality. TRINIDAD \& TOBAGO, St. Augustine. 

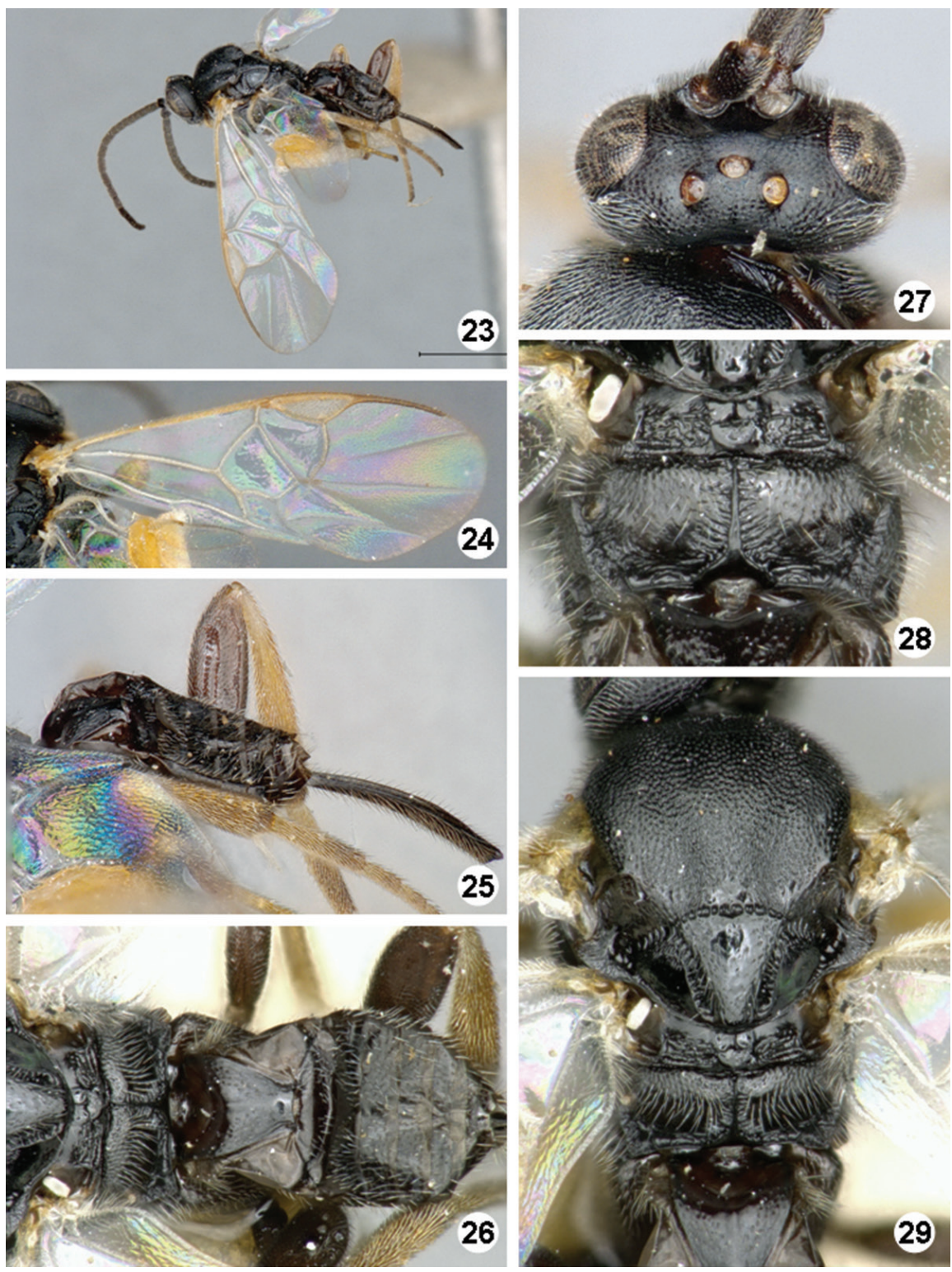

Figures 23-29. Iconella isolata. 23 Habitus, dorso-lateral view 24 Fore wing 25 Ovipositor sheats, mesofermur, and metatibia $\mathbf{2 6}$ Propodeum and metasoma, dorsal view 27 Head, dorsal view 28 Propodeum 29 Mesosoma and mediotergite I dorsal view.

Holotype. + , NMNH (not examined). Paratypes. $2 q$ and $1 \partial^{\lambda}, \mathrm{CNC}$ (examined). Specimens examined. 11 , $5 \hat{\jmath}$ (CNC) British Guiana, Cayman islands (Grand Cayman, Georgetown), Grenada, Montserrat, Saint Kitts \& Nevis (Nevis, Round Hill), Trinidad \& Tobago (Paradise Mt., St. Augustine, and Tacarigua). Collecting dates of specimens examined: February, March and December (1950-1965). 
Description. Promefur color: mostly yellow, dark brown area limited to anterior 0.2 or less, rarely dark brown on anterior half, yellow on posterior half. Meso- and meta-femur color: mostly dark brown but with proximal $0.1-0.2 \times$ yellow to orange. Metatibia and metatarsus color: Metatibia mostly yellow, at most with very small and faint brown spot on posterior $0.1 \times$ or less; metatarsus mostly yellow, except for brown area on posterior half of first tarsomerus. Tegula and humeral complex color: tegula and humeral complex fully yellow to yellowish-white (Fig. 29). Pterostigma color: centrally transparent, with yellow-white margins (Fig. 24). Fore wing veins color: most of veins transparent or at most yellowish-white, margins of same color than interior of vein. Body length (head to apex of metasoma): $2.4 \mathrm{~mm}, 2.5 \mathrm{~mm}$, $2.6 \mathrm{~mm}, 2.7 \mathrm{~mm}, 2.8 \mathrm{~mm}$ or $2.9 \mathrm{~mm}$. Fore wing length: $2.8 \mathrm{~mm}, 2.9 \mathrm{~mm}, 3.0 \mathrm{~mm}, 3.1 \mathrm{~mm}$ or $3.2 \mathrm{~mm}$. Ocular-ocellar linelposterior ocellus diameter: $1.9 \times$ or $2.0 \times$. Interocellar distancel posterior ocellus diameter: $1.9 \times, 2.0 \times$ or $2.1 \times$ (Fig. 27). Antennal flagellomere 2 length/width: $2.2 \times, 2.3 \times$ or $2.5 \times$. Antennal flagellomere 14 length/width: $1.3 \times$ or $1.4 \times$. Length of flagellomere 2/length of flagellomere 14: $2.3 \times, 2.4 \times$ or $2.5 \times$. Metafemur length/width: $3.2 \times$ or 3.3 $\times$. Mesoscutellar disc: mostly smooth with few, scattered punctures near margins (Fig. 29). Number of pits in scutoscutellar sulcus: usually 12 or less, ocasionally reaching up to 14 pits. Propodeum background sculpture: anterior 0.2-0.4 $\times$ with fine puntures; posterior 0.6-0.8 $\times$ mostly smooth, at most with some small carinae (mostly radiating from strong, longitudinal median carina) (Fig. 28). Mediotergite 1 width at anterior margin/width at posterior margin: $2.1 \times, 2.6 \times$ or $2.8 \times$. Mediotergite 2 width at posterior margin/length: $3.6 \times, 3.7 \times, 3.8 \times, 4.5$ $\times$ or $4.6 \times$ (Fig. 26). Ovipositor sheaths length/metatibial length: $1.0 \times($ Fig. 25$)$.

Male. As female.

Molecular data. No DNA barcode sequence was available for this species, the only one among the New World species without molecular data.

Biology/ecology. Host: Ancylostomia stercorea (Pyralidae).

Distribution. British Virgin Islands, Cayman Islands, Dominica, Grenada, Guyana, Montserrat, Puerto Rico, Saint Kitts \& Nevis, Trinidad \& Tobago.

Comments. Until now, $I$. isolata had been considered to be a subspecies of $I$. etiellae. After study of numerous specimens, we have found consistent and significant differences in morphology, hosts and geographical distribution and thus consider $I$. isolata as a different species on its own. Cayman Islands and Saint Kitts \& Nevis are new locality records, based on CNC specimens. The specimen from Cayman Islands represents the westernmost locality, and it expands considerably the previously known distribution of the species in the Caribbean islands.

\section{Iconella jayjayrodriguezae Fernández-Triana, sp. n.} http://zoobank.org/A1E2E3FC-6C2D-4E6F-83C0-AD388D55DCB5 http://species-id.net/wiki/Iconella_jayjayrodriguezae Figure 30-36

Type locality. COSTA RICA, Alajuela, Area de Conservacion Guanacaste, Sector Rincon Rain Forest, Sendero Venado, 420m. Lat: 10.89678, Long: -85.27001. 

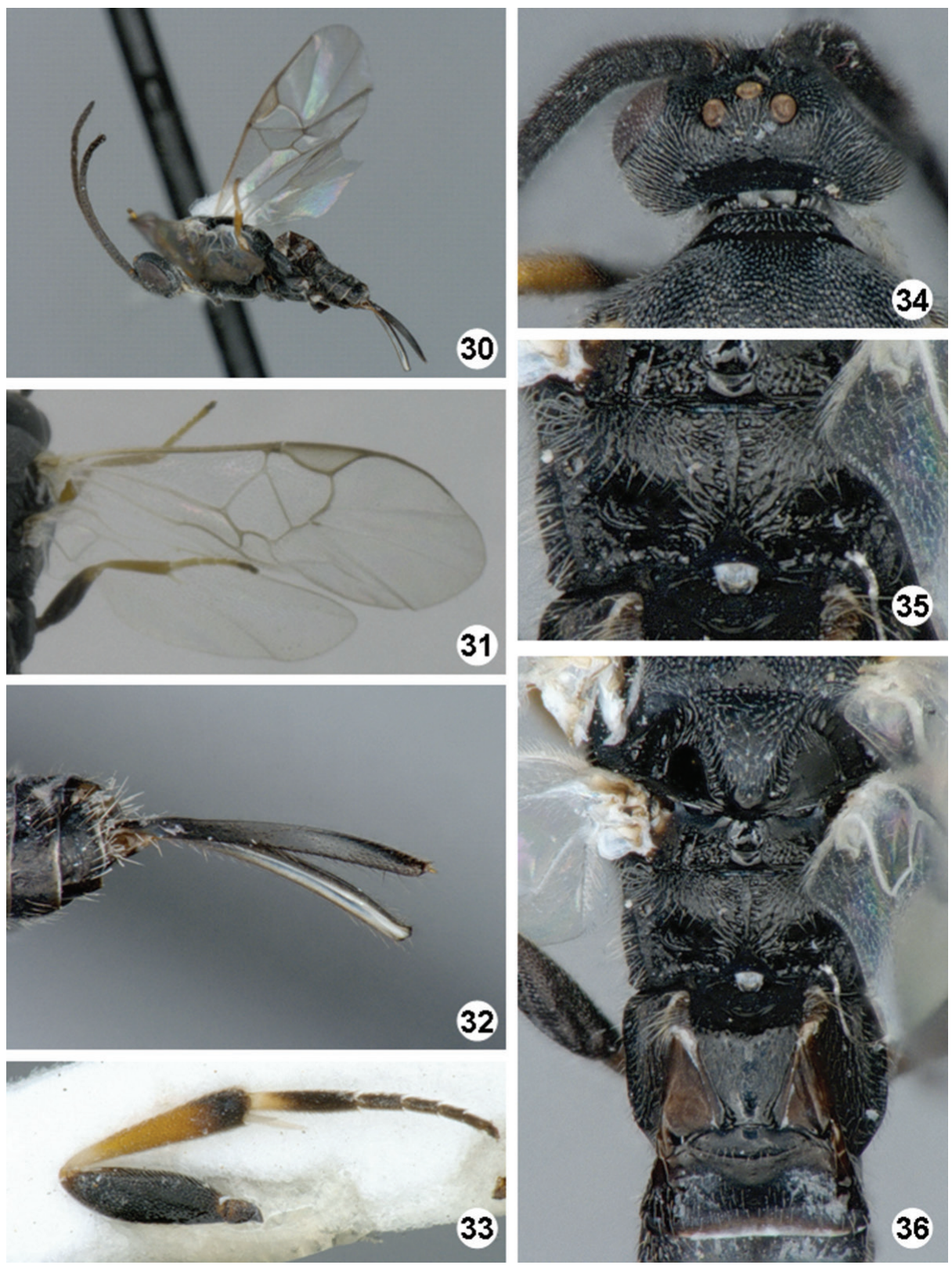

Figures 30-36. Iconella jayjayrodriguezae. $\mathbf{3 0}$ Habitus, lateral view $\mathbf{3}$ I Fore wing $\mathbf{3 2}$ Ovipositor sheaths 33 Hind leg 34 Head, dorsal view 35 Propodeum 36 Meso- and metasoma (partially), dorsal view.

Holotype. , NMNH. First label: DHJPAR0039740. Second label: Voucher: D.H.Janzen \& W.Hallwachs, DB: http://janzen.sas.upenn.edu, Area de Conservacion Guanacaste, COSTA RICA, 09-SRNP-41791. Collecting date of caterpillar host 21.vii.2009, collection date (eclosion date) of wasp 10.viii.2009. 
Specimens examined. Paratypes: 3 †, 1 ○े (CNC) Costa Rica, Alajuela, ACG, Sector San Cristobal, Rio Blanco Abajo, 500m, Lat: 10.90037 Long: -85.37254; 1 q (CNC) Mexico, Chiapas, $16^{\circ} 58^{\prime} \mathrm{N}, 91^{\circ} 47^{\prime} \mathrm{W}, 23-25 . v i i i .1978$. Collecting dates of specimens examined: January, March, April, June, July, and September (2009-2011) for Costa Rican specimens; August (1978) for Mexican specimen.

Description. Promefur color: mostly yellow, dark brown area limited to anterior 0.2 or less. Meso- and meta-femur color: mostly dark brown but with proximal 0.1-0.2 × yellow to orange. Metatibia and metatarsus color: Metatibia with brown to black coloration on posterior $0.2-0.4 \times$; metatarsus mostly dark brown, except for yellowish area on anterior half of first tarsomerus (Fig. 33). Tegula and humeral complex color: tegula and humeral complex fully yellow to yellowish-white. Pterostigma color: centrally transparent, with yellow-white margins, rarely centrally yellow-white, with thin brown margins (Fig. 31). Fore wing veins color: at least some veins with thin brown margins and interior of veins yellow to light brown. Body length (head to apex of metasoma): $2.9 \mathrm{~mm}$ or 3.0 $\mathrm{mm}$. Fore wing length: $3.2 \mathrm{~mm}$ or $3.3 \mathrm{~mm}$. Ocular-ocellar linelposterior ocellus diameter: $2.0 \times$. Interocellar distancelposterior ocellus diameter: $1.9 \times$ (Fig. 34). Antennal flagellomere 2 length/width: $2.3 \times, 2.4 \times$ or $2.5 \times$. Antennal flagellomere 14 length/width: $1.3 \times$, rarely $1.1 \times$ or $1.5 \times$. Length of flagellomere 2/length of flagellomere 14: $2.3 \times, 2.4 \times, 2.5 \times$ or 2.6 $\times$. Metafemur length/width: $3.2 \times, 3.3 \times$ or $3.4 \times$. Mesoscutellar disc: mostly smooth with few, scattered punctures near margins (Fig. 36). Number of pits in scutoscutellar sulcus: usually 12 or less, ocasionally reaching up to 14 pits. Propodeum background sculpture: anterior $0.2-0.4 \times$ with rather dull puntures; posterior $0.6-0.8 \times$ mostly sculptured, with mix of small puntures and carinae (mostly radiating from strong, longitudinal median carina) (Fig. 35). Mediotergite 1 width at anterior margin/width at posterior margin: $3.1 \times, 3.2 \times$ or $3.3 \times$. Mediotergite 2 width at posterior margin/length: $3.7 \times, 3.8 \times, 3.9$ $\times$ or $4.1 \times$ (Fig. 36). Ovipositor sheaths length/metatibial length: $1.1 \times($ Fig. 32).

Male. As female.

Molecular data. We analyzed eight 650-658 bp barcodes for this species, one from Mexico and seven from Costa Rica.

Biology/ecology. Host: An undescribed species of Spilomelinae (Crambidae) with provisional name "spiloBioLep01 BioLep414" in the ACG database (http://janzen.sas. upenn.edu/caterpillars/database.lasso). Caterpillar collected while feeding on the foliage of Lepidoploa salzmannii and Lepidoploa tortuosa (Asteraceae).

Distribution. Costa Rica (ACG), and Mexico (Chiapas). In ACG it has been reared from four rainforest localities between $420-500 \mathrm{~m}, 2-10 \mathrm{~km}$ apart, in six different months.

Comments. Although morphologically similar to Iconella andydeansi, I. jayjayrodriguezae is known from two very widely separated places in Central America, and the barcoding sequences of the two species differ by $8.7 \%$ ( $57 \mathrm{bp}$ ).

Etymology. This species is named in honor of Josephine J. Rodriguez (National Center for Ecological Analysis and Synthesis, University of California, Santa Barbara, United States) in recognition of her outstanding enthusiasm for studying the taxonomy and biology of the microgastrine wasps of ACG (e.g., Smith et al. 2008). 


\section{Acknowledgements}

We gratefully acknowledge the support of the Guanacaste Dry Forest Conservation Fund, the Wege Foundation, the International Conservation Fund of Canada, the JRS Biodiversity Foundation, Jessie Hill, and the University of Pennsylvania for funding portions of the research. JFT gratefully acknowledge the support and sponsoring of the Lucid team (http://www.lucidcentral.com/). This study was also supported by NSF DEB 0515699 to DHJ and by a Natural Sciences and Engineering Research Council of Canada (NSERC) Discovery Grant to MAS. Laboratory analyses of these sequences were funded by the Government of Canada through Genome Canada and the Ontario Genomics Institute (2008-0GI-ICI-03). Kees van Achterberg (Naturalis, Leiden) and an anonymous reviewer helped to improve the quality of the manuscript.

\section{References}

Achterberg C van (2002) Western Palaearctic genera of the subfamily Microgastrinae: a reappraisal of the generic and tribal division (Hymenoptera: Braconidae). In: Melika G, Thuróczy G (Eds) Parasitic wasps: evolution, systematics, biodiversity and biological control. Agroinform, Budapest, Hungary, 19-35.

Bartlett BR, Clausen CP, DeBach P, Goeden RD, Legner EF, McMurtry JA, Oatman ER (1978) Introduced parasites and predators of arthropod pests and weeds: a world review Agriculture Handbook No. 480, Agricultural Research Service, United States Department of Agriculture, Washington, District of Columbia.

Bennett FD (1960) Parasites of Ancylostomia stercorea (Zell.) (Pyralidae, Lepidoptera), a pod borer attacking pigeon pea in Trinidad. Bulletin of Entomological Research 50: 737-757. doi: $10.1017 /$ S0007485300054778

Broad GR, Shaw MR, Godfray HCJ (2012) Checklist of British and Irish Braconidae (Hymenoptera). http://www.nhm.ac.uk/resources-rx/files/braconidae-checklist-final-34139.pdf [accessed 11.III.2013]

Darriba D, Taboada GL, Doallo R, Posada D (2012) jModelTest 2: more models, new heuristics and parallel computing. Nature Methods 9: 772. doi: 10.1038/nmeth.2109

Deans AR, Whitfield JB, Janzen DH (2003) Taxonomy and natural history of the microgastrine genus Alphomelon Mason (Hymenoptera: Braconidae). Journal of Hymenoptera Research 12(1): 1-41.

Drummond AJ, Ashton B, Buxton S, Cheung M, Cooper A, Duran C, Field M, Heled J, Kearse M, Markowitz S, Moir R, Stones-Havas S, Sturrock S, Thierer T, Wilson A (2011) Geneious v6.0.5 http://www.geneious.com

Fernández-Triana J (2010) Eight new species and an annotated checklist of Microgastrinae (Hymenoptera: Braconidae) from Canada and Alaska. ZooKeys 63: 1-53. doi: 10.3897/ zookeys.63.565

Fernández-Triana J, Smith MA, Bodreault C, Goulet H, Hebert PDN, Smith AC, Roughley R (2011) A poorly known high-latitude parasitoid wasp community: unexpected diver- 
sity and dramatic changes through time. PLoS ONE 6(8): e23719. doi: 10.1371/journal. pone.0023719

Goloboff PA, Farris JS, Nixon KC (2008) TNT, a free program for phylogenetic analysis. Cladistics. 24: 774-786. doi: 10.1111/j.1096-0031.2008.00217.x

Hajibabaei M, Smith MA, Janzen DH, Rodriguez JJ, Whitfield JB, Hebert PDN (2006) A minimalist barcode can identify a specimen whose DNA is degraded. Molecular Ecology Notes 6: 959-964. doi: 10.1111/j.1471-8286.2006.01470.x

Huber JT, Sharkey MJ (1993) Structure. In: Goulet H, Huber JT (Eds) Hymenoptera of the world: an identification guide to families. Agriculture Canada Research Branch, Monograph No. 1894E, Ottawa, Canada, 13-59.

Ivanova NV, Dewaard JR, Hebert PDN (2006) An inexpensive, automation-friendly protocol for recovering high-quality DNA. Molecular Ecology Notes 6 (4): 998-1002. doi: 10.1111/j.1471-8286.2006.01428.x

Janzen DH, Hallwachs W, Blandin P, Burns JM, Cadiou J, Chacon I, Dapkey T, Deans AR, Epstein ME, Espinoza B, Franclemont JG, Haber WA, Hajibabaei M, Hall JPW, Hebert PDN, Gauld ID, Harvey DJ, Hausmann A, Kitching I, Lafontaine D, Landry J, Lemaire C, Miller JY, Miller JS, Miller L, Miller SE, Montero J, Munroe E, Rab Green S, Ratnasingham S, Rawlins JE, Robbins RK, Rodriguez JJ, Rougerie R, Sharkey MJ, Smith MA, Solis MA, Sullivan JB, Thiaucourt P, Wahl DB, Weller SJ, Whitfield JB, Willmott KR, Wood DM, Woodley NE, Wilson JJ (2009) Integration of DNA barcoding into an ongoing inventory of complex tropical biodiversity. Molecular Ecology Resources 9 (Supplement 1): 1-26. doi: 10.1111/j.1755-0998.2009.02628.x

Mason WRM (1981) The polyphyletic nature of Apanteles Foerster (Hymenoptera: Braconidae): A phylogeny and reclassification of Microgastrinae. Memoirs of the Entomological Society of Canada 115: 1-147. doi: 10.4039/entm113115fv

Meusnier I, Singer GAC, Landry JF, Hickey DA, Hebert PD, Hajibabaei M (2008) A universal DNA mini-barcode for biodiversity analysis. BMC Genomics 9: 214. doi: 10.1186/14712164-9-214

Muesebeck CFW (1955) New reared Braconidae from Trinidad (Hymenoptera). Proceedings of the Entomological Society of Washington 57(4): 161-164.

Nixon G (1965) A reclassification of the tribe Microgasterini (Hymenoptera: Braconidae). Bulletin of the British Museum (Natural History), Entomology series, Supplement 2: 1-284.

Rambaut A, Drummond AJ (2009) Tracer v1.5. http://beast.bio.ed.ac.uk/Tracer

Ronquist F, Huelsenbeck JP (2003) MRBAYES 3: Bayesian phylogenetic inference under mixed models. Bioinformatics 19: 1572-1574. doi: 10.1093/bioinformatics/btg180

Saitou N, Nei M (1987) The neighbor-joining method: a new method for reconstructing phylogenetic trees. Molecular Biology and Evolution 4: 406-425.

Shorthouse DP (2010) SimpleMappr, an online tool to produce publication-quality point maps. http://www.simplemappr.net [accessed 13 March, 13]

Smith MA, Woodley NE, Janzen DH, Hallwachs W, Hebert PDN (2006) DNA barcodes reveal cryptic host-specificity within the presumed polyphagous members of a genus of parasitoid flies (Diptera: Tachinidae). Proceedings of the National Academy of Sciences 103: 3657-3662. doi: 10.1073/pnas.0511318103 
Smith MA, Wood DM, Janzen DH, Hallwachs W, Hebert PDN (2007) DNA barcodes affirm that 16 species of apparently generalist tropical parasitoid flies (Diptera, Tachinidae) are not all generalists. Proceedings of the National Academy of Sciences 104: 4967-4972. doi: 10.1073/pnas.0700050104

Smith MA, Rodriguez JJ, Whitfield JB, Deans AR, Janzen DH, Hallwachs W, Hebert PDN (2008) Extreme diversity of tropical parasitoid wasps exposed by iterative integration of natural history, DNA barcoding, morphology, and collections. Proceedings of the National Academy of Sciences 105: 12359-12364. doi: 10.1073/pnas.0805319105

Smith MA, Fernández-Triana JL, Eveleigh E, Gómez J, Guclu C, Hallwachs W, Hebert PDN, Hrcek J, Huber JT, Janzen DH, Mason PG, Miller SE, Quicke DLJ, Rodriguez JJ, Rougerie R, Shaw MR, Varkonyi G, Ward D, Whitfield JB, Zaldívar-Riverón A (2013) DNA barcoding and the taxonomy of Microgastrinae wasps (Hymenoptera, Braconidae): impacts after 8 years and nearly 20000 sequences. Molecular Ecology Resources, 13: 168176. doi: 10.1111/1755-0998.12038

Stamatakis A (2006) RAxML-VI-HPC: maximum likelihotod-based phylogenetic analyses with housands of taxa and mixed models. Bioinformatics, 22(21): 2688-2690. doi: 10.1093/bioinformatics/btl446

Tamura K, Nei M (1993) Estimation of the number of nucleotide substitutions in the control region of mitochondrial DNA in humans and chimpanzees. Molecular Biology and Evolution 10: $512-526$.

Viereck HL (1911) Descriptions of six new genera and thirty-one new species of Ichneumon flies. Proceedings of the United States National Museum 40(1812): 173-196.

Whitfield JB (1997) Subfamily Microgastrinae. In: Wharton RA, Marsh PM, Sharkey MJ (Eds) Manual of the New World genera of the family Braconidae (Hymenoptera). Special Publication No. 1 International Society of Hymenopterists, Washington, D.C., 333-364. Yu DSK, van Achterberg C, Horstmann K (2012) Taxapad 2012, Ichneumonoidea 2011. Database on flash-drive. www.taxapad.com, Ottawa, Ontario, Canada. 


\section{Appendix}

Lucid key to of the New World species of the parasitoid wasp Iconella (Hymenoptera, Braconidae, Microgastrinae). (doi: 10.3897/zookeys.321.5160.app) File format: Lucid Key Data (lk4).

Copyright notice: This dataset is made available under the Open Database License (http://opendatacommons.org/licenses/odbl/1.0/). The Open Database License $(\mathrm{ODbL})$ is a license agreement intended to allow users to freely share, modify, and use this Dataset while maintaining this same freedom for others, provided that the original source and author(s) are credited.

Citation: Fernández-Triana JL, Cardinal S, Whitfield JB, Hallwachs W, Smith AM, Janzen DH (2013) A review of the New World species of the parasitoid wasp Iconella (Hymenoptera, Braconidae, Microgastrinae). ZooKeys 321: 65-87. doi: 10.3897/zookeys.321.5160 Lucid key to of the New World species of the parasitoid wasp Iconella (Hymenoptera, Braconidae, Microgastrinae). ZooKeys 321: 65-87. doi: 10.3897/zookeys.321.5160.app 